\title{
Single cell analysis of highly metastatic circulating tumor cells by combining a self-folding induced release reaction with a cell capture microchip
}

Chang Feng, ${ }^{a, b, \#}$ Dongsheng Mao, ${ }^{a, \#}$ Cuicui Lu, ${ }^{a}$ Qianqian Zhang, ${ }^{a}$ Xiaohao Liu,,${ }^{a, d}$

Qirui Wu, ${ }^{c}$ Xiuqing Gong, ${ }^{c}$ Guifang Chen, ${ }^{a} *$ Xiaoli $Z h u^{a, *}$

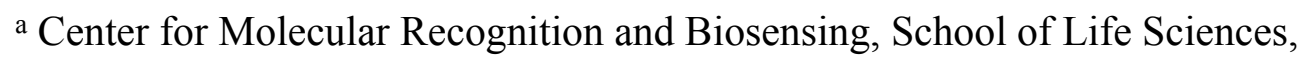

Shanghai University, Shanghai 200444, P. R. China.

b School of Medicine, Shanghai University, Shanghai 200444, P. R. China.

${ }^{c}$ Materials Genome Institute, Shanghai University, Shanghai 200444, P. R. China.

d Shanghai Tenth People's Hospital, School of Medicine, Tongji University, Shanghai 200092, P. R. China.

\# These authors contributed equally to this work.

*Corresponding Authors: gfchen@shu.edu.cn; xiaolizhu@shu.edu.cn

\section{Table of Contents}

Table S1. Sequences of DNA oligonucleotides used in this work

Table S2. The cell viability for evaluating drug sensitivity of different tumor cells with 
Figure S1. Electrophoresis characterization of exponential sFiR related DNA oligonucleotides when toehold is $0 \mathrm{nt}$ and $5 \mathrm{nt}$.

Figure S2. Optimization of the ratio of nicking enzyme and polymerase.

Figure S3. Quantitative analysis of primer by linear sFiR.

Figure S4. Quantitative analysis of primer by exponential sFiR.

Figure S5. Performance of exponential sFiR in CutSmart and PBS.

Figure S6. Fluorescence signal variation of exponential sFiR in equilibrium buffer and medium with different concentration of magnesium ions.

Figure S7. Immunofluorescence of JUP in three cell lines.

Figure S8. Crosslinking efficiency between the antibody and the primer.

Figure S9. Apoptosis analysis of different cells before and after immuno-sFiR analysis.

Figure S10. Morphology of HeLa cells after immuno-sFiR analysis.

Figure S11. Cell viability analysis of HeLa cells after immuno-sFiR analysis.

Figure S12. Cell viability analysis of HeLa cells with immuno-sFiR and continued culture after the analysis.

Figure S13. JUP expression before and after immuno-sFiR analysis.

Figure S14. Standard curve at different concentration of HeLa cells using MTT assay. 
Table S1. Sequences of DNA oligonucleotides used in this work

\begin{tabular}{|c|c|c|c|}
\hline \multicolumn{2}{|c|}{ oligonucleotide } & Sequence $\left(5^{\prime}-3^{\prime}\right)$ & Modification \\
\hline \multirow{6}{*}{ Linear sFiR } & $\mathrm{L}_{0}$-Tem & $\begin{array}{l}\text { GCGTCTCAGTCTCTGCCTCAGCACCC } \\
\text { TAGTCACACTCCAATATAAAAAATG } \\
\text { AGGCAGAGACTGAGACGC }\end{array}$ & 3'-C3 Spacer \\
\hline & $\mathrm{L}_{0}-\mathrm{S}$ & TGAGGCAGAGACTGAGACGC & \\
\hline & $\mathrm{L}_{5}$-Tem & $\begin{array}{l}\text { GCGTCTCAGTCTCTGTCTGGCCTCAG } \\
\text { CACCCTAGTCACACTCCAATATACCA } \\
\text { GACAGAGACTGAGACGC }\end{array}$ & 3'-C3 Spacer \\
\hline & $\mathrm{L}_{5}-\mathrm{S}$ & TGAGGCCAGACAGAGACTGAGACGC & \\
\hline & $\mathrm{L}-\mathrm{P}_{22}$ & TATTGGAGTGTGACTAGGGTGC & \\
\hline & $\mathrm{L}-\mathrm{P}_{10}$ & ACTAGGGTGC & \\
\hline \multirow{7}{*}{$\begin{array}{l}\text { Exponential } \\
\text { sFiR }\end{array}$} & $\mathrm{E}_{0}$-Tem & $\begin{array}{l}\text { GCGTCTCAGTCTCTGCCTCAGCGTCT } \\
\text { CAGTAACACACCAATATATTTTTTGA } \\
\text { GGCAGAGACTGAGACGC }\end{array}$ & 3'-C3 Spacer \\
\hline & $\mathrm{E}_{0}-\mathrm{S}$ & TGAGGCAGAGACTGAGACGC & \\
\hline & $\mathrm{E}_{5}$-Tem & $\begin{array}{l}\text { GCGTCTCAGTCTCTGTCTGGCCTCAG } \\
\text { CGTCTCAGTAACACACCAATATACC } \\
\text { AGACAGAGACTGAGACGC }\end{array}$ & 3'-C3 Spacer \\
\hline & $\mathrm{E}_{5}-\mathrm{S}$ & TGAGGCCAGACAGAGACTGAGACGC & \\
\hline & $\mathrm{E}-\mathrm{P}_{22}$ & TATTGGTGTGTTACTGAGACGC & 5'-SH \\
\hline & E-P 10 & ACTGAGACGC & \\
\hline & $\begin{array}{l}\text { E-P } 22^{-} \\
\text {FAM }\end{array}$ & TATTGGTGTGTTACTGAGACGC & $\begin{array}{l}\text { 5'-SH } \\
\text { 3'-FAM }\end{array}$ \\
\hline \multirow[t]{2}{*}{ EXPAR } & Tem & $\begin{array}{l}\text { GCGTCTCAGTCTCTGCCTCAGCGTCT } \\
\text { CAGTAACACACCAATA }\end{array}$ & 3'-C3 Spacer \\
\hline & Pri & TATTGGTGTGTTACTGAGACGC & \\
\hline \multicolumn{2}{|c|}{ Molecular beacon-MB } & $\begin{array}{l}\text { GGTTGGGCGTCTCAGTCTCTGCCAAC } \\
\text { C }\end{array}$ & $\begin{array}{l}\text { 5'-FAM } \\
\text { 3'-BHQ1 }\end{array}$ \\
\hline \multicolumn{2}{|c|}{ MUC1 aptamer } & $\begin{array}{l}\text { (T)5GCAGTTGATCCTTTGGATACCCT } \\
\text { GG }\end{array}$ & $5^{\prime}-\mathrm{NH} 2$ \\
\hline
\end{tabular}


Table S2. The cell viability for evaluating drug sensitivity of different tumor cells with and without immuno-sFiR analysis (\%).

\begin{tabular}{|c|c|c|c|c|c|c|c|c|c|}
\hline & $\begin{array}{l}\text { Doxorub } \\
\text { icin }(\mu \mathrm{M})\end{array}$ & 0 & 0.1 & 0.2 & 0.4 & 0.8 & 1.6 & 3.2 & 6.4 \\
\hline \multirow{3}{*}{$\begin{array}{l}\text { Immuno- } \\
\text { sFiR (w/o) }\end{array}$} & $\mathrm{HeLa}$ & 100.00 & 87.56 & 91.18 & 81.52 & 43.33 & 14.99 & 11.13 & 13.03 \\
\hline & $\begin{array}{l}\text { MDA- } \\
\text { MB-231 }\end{array}$ & 100.00 & 88.94 & 70.07 & 58.32 & 57.55 & 45.52 & 37.64 & 37.49 \\
\hline & MCF-7 & 100.00 & 78.94 & 68.90 & 63.06 & 55.45 & 41.21 & 25.96 & 18.68 \\
\hline \multirow{5}{*}{$\begin{array}{l}\text { Immuno- } \\
\text { sFiR (with) }\end{array}$} & $\mathrm{HeLa}$ & 100.00 & 86.54 & 97.42 & 79.40 & 38.82 & 15.10 & 11.61 & 13.09 \\
\hline & $\begin{array}{l}\text { MDA- } \\
\text { MB-231 }\end{array}$ & 100.00 & 92.46 & 72.26 & 56.96 & 50.46 & 44.13 & 41.51 & 39.23 \\
\hline & MCF-7 & 100.00 & 88.94 & 78.40 & 68.84 & 60.37 & 44.67 & 30.71 & 22.48 \\
\hline & Gemcita & & & & & & & & \\
\hline & $\begin{array}{l}\text { bine } \\
(\mu \mathrm{M})\end{array}$ & 0 & 0.25 & 0.5 & 1.0 & 2.0 & 4.0 & 8.0 & 16.0 \\
\hline \multirow{3}{*}{$\begin{array}{l}\text { Immuno- } \\
\text { sFiR (w/o) }\end{array}$} & HeLa & 100.00 & 97.28 & 96.88 & 95.77 & 94.90 & 94.18 & 92.86 & 90.36 \\
\hline & $\begin{array}{l}\text { MDA- } \\
\text { MB-231 }\end{array}$ & 100.00 & 99.88 & 98.39 & 97.31 & 95.90 & 95.25 & 93.38 & 92.91 \\
\hline & MCF-7 & 100.00 & 98.75 & 96.93 & 97.34 & 94.77 & 92.63 & 92.51 & 91.38 \\
\hline \multirow{5}{*}{$\begin{array}{l}\text { Immuno- } \\
\text { sFiR (with) }\end{array}$} & $\mathrm{HeLa}$ & 100.00 & 96.04 & 98.10 & 99.62 & 97.38 & 95.95 & 91.90 & 91.74 \\
\hline & $\begin{array}{l}\text { MDA- } \\
\text { MB-231 }\end{array}$ & 100.00 & 97.04 & 99.34 & 96.31 & 97.10 & 95.90 & 93.97 & 92.79 \\
\hline & MCF-7 & 100.00 & 96.16 & 95.73 & 95.39 & 93.55 & 93.16 & 92.07 & 91.36 \\
\hline & Paclitaxe & & & & & & & & \\
\hline & $\begin{array}{l}\text { l } \\
(\mu M)\end{array}$ & 0 & 0.49 & 0.99 & 1.98 & 3.97 & 7.94 & 15.88 & 31.76 \\
\hline \multirow{3}{*}{$\begin{array}{l}\text { Immuno- } \\
\text { sFiR (w/o) }\end{array}$} & $\mathrm{HeLa}$ & 100.00 & 83.68 & 64.05 & 51.07 & 37.04 & 26.21 & 8.13 & 9.60 \\
\hline & $\begin{array}{l}\text { MDA- } \\
\text { MB-231 }\end{array}$ & 100.00 & 97.77 & 89.04 & 85.32 & 84.00 & 76.72 & 68.60 & 59.35 \\
\hline & MCF-7 & 100.00 & 52.08 & 49.19 & 45.35 & 45.95 & 41.35 & 41.05 & 40.72 \\
\hline \multirow{3}{*}{$\begin{array}{c}\text { Immuno- } \\
\text { sFiR (with) }\end{array}$} & $\mathrm{HeLa}$ & 100.00 & 86.05 & 82.19 & 60.62 & 33.79 & 17.48 & 9.11 & 8.18 \\
\hline & $\begin{array}{l}\text { MDA- } \\
\text { MB-231 }\end{array}$ & 100.00 & 98.36 & 92.83 & 91.18 & 79.11 & 76.73 & 70.14 & 60.12 \\
\hline & MCF-7 & 100.0 & 53.02 & 51.24 & 47.29 & 46.75 & 44.61 & 43.17 & 42.62 \\
\hline
\end{tabular}



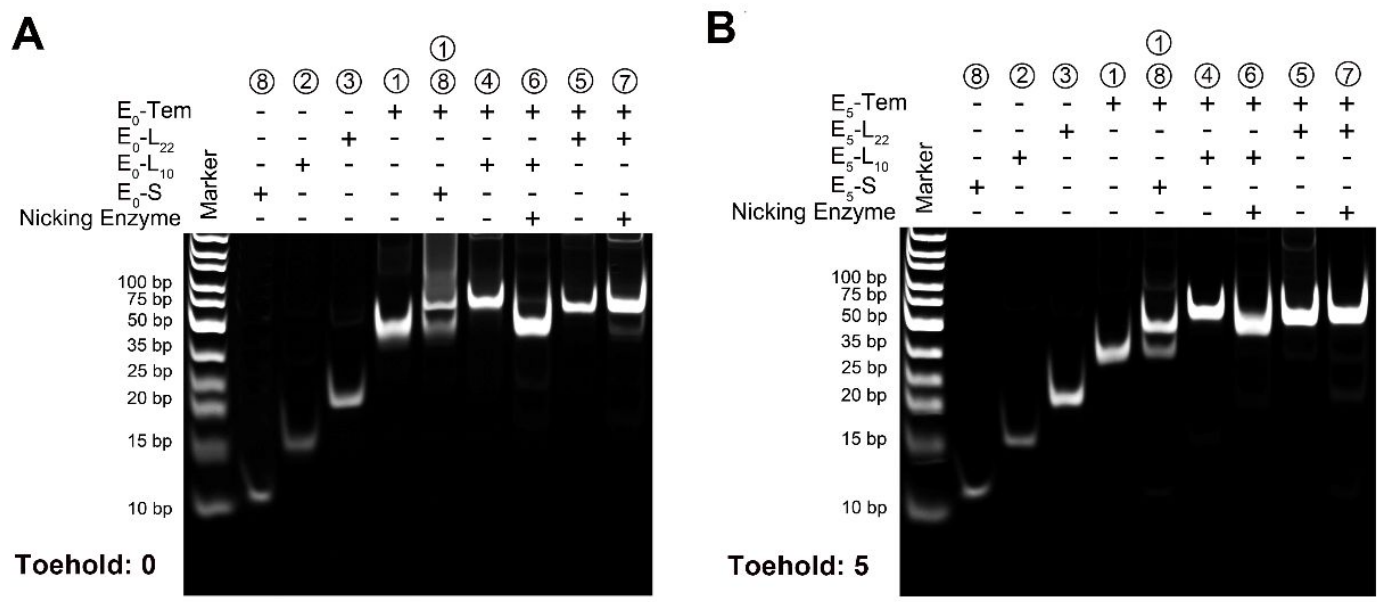

Figure S1. Electrophoresis characterization of exponential sFiR related DNA oligonucleotides when toehold is $0 \mathrm{nt}$ and $5 \mathrm{nt}$.

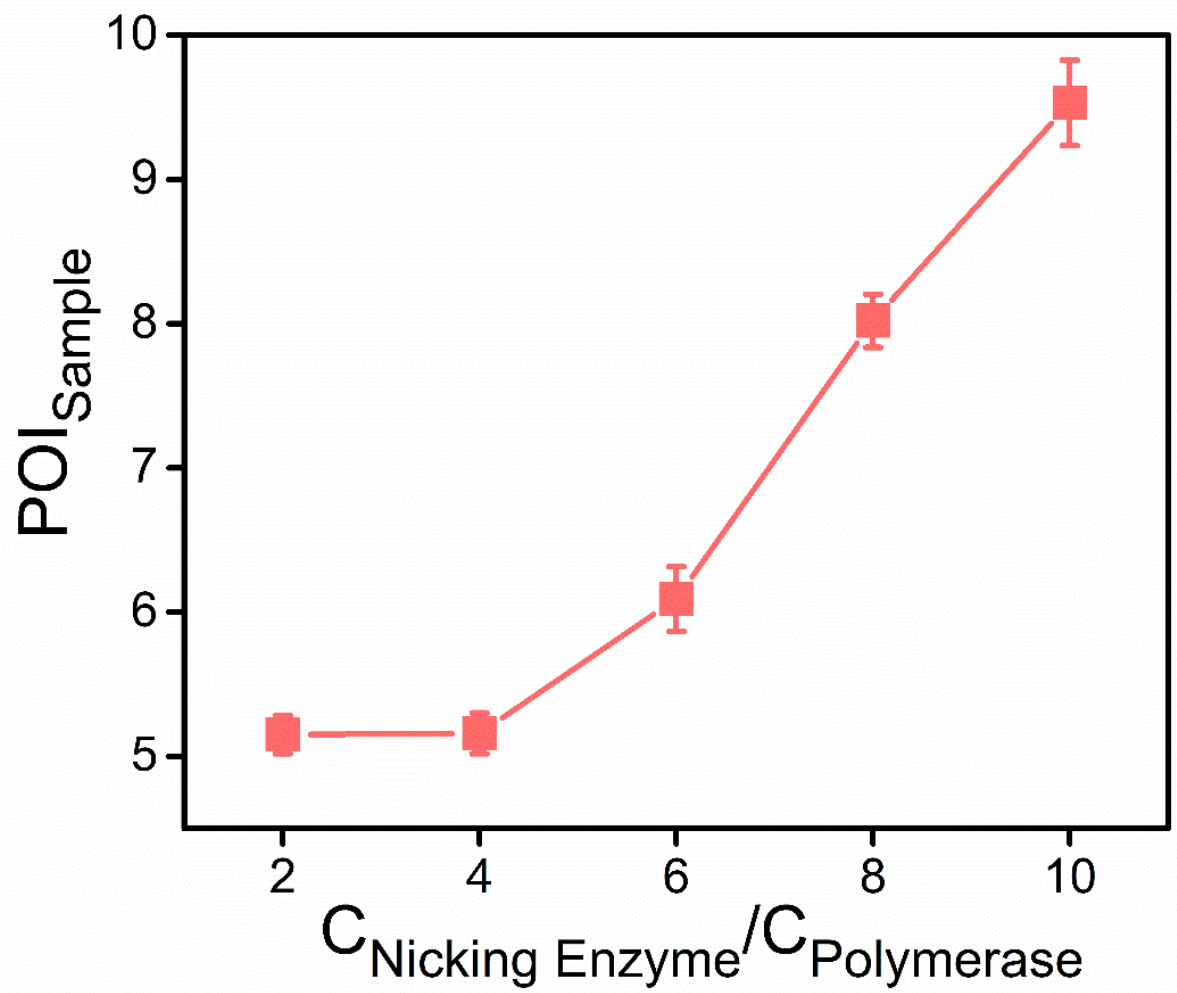

Figure S2. Optimization of the ratio of nicking enzyme and polymerase. Error bars represent the standard deviation of three experiments. 


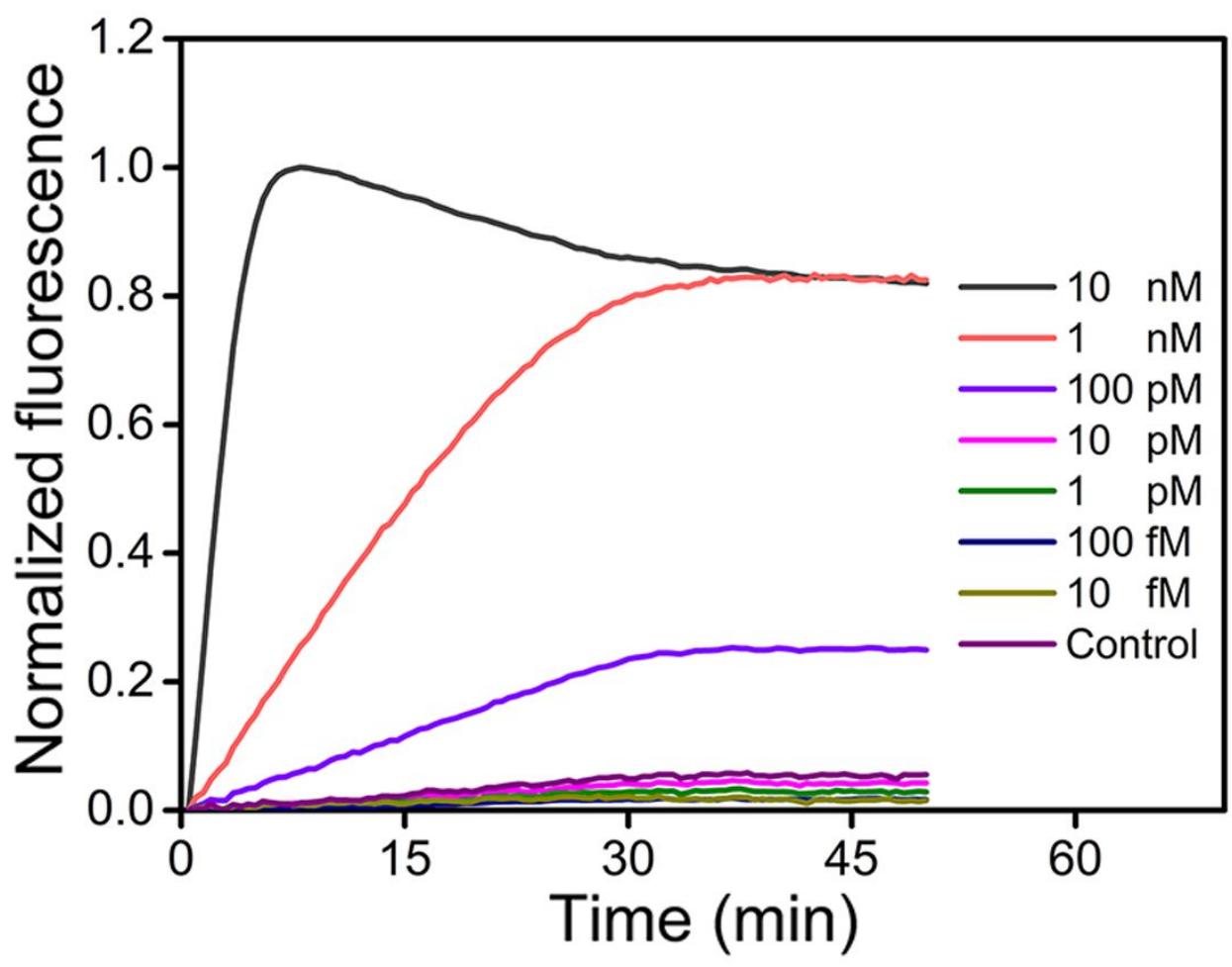

Figure S3. Quantitative analysis of primer by linear sFiR.

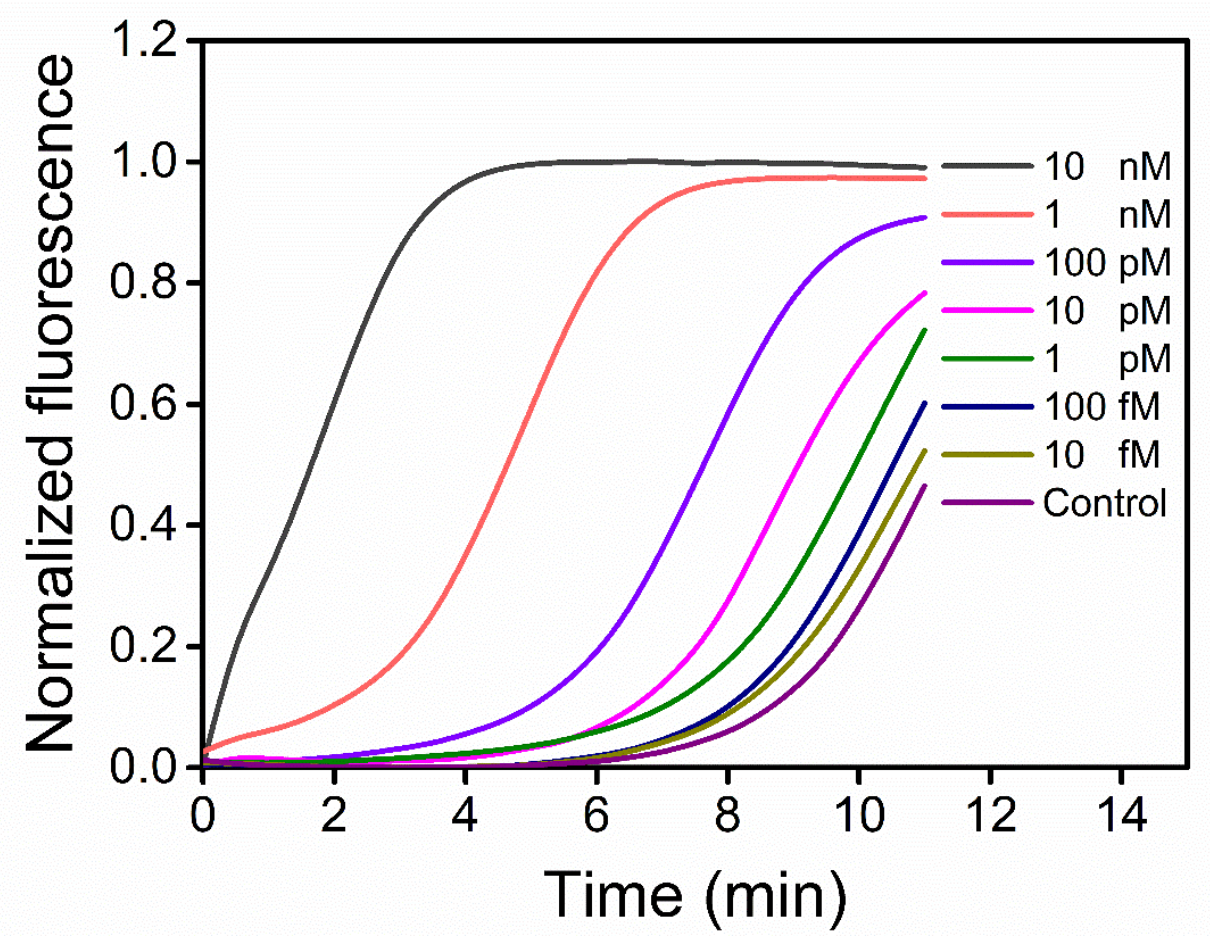

Figure S4. Quantitative analysis of primer by exponential sFiR. 


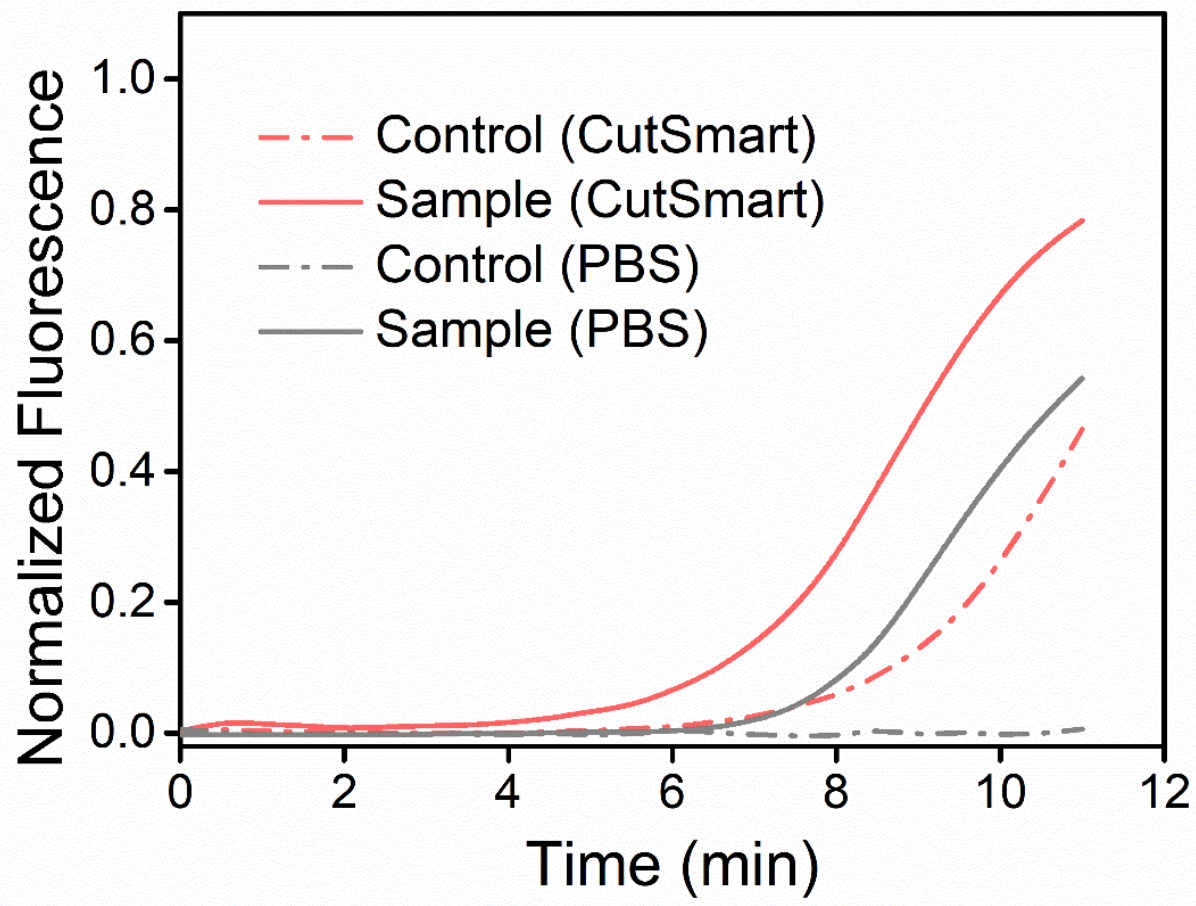

Figure S5. Performance of exponential sFiR in CutSmart and PBS.
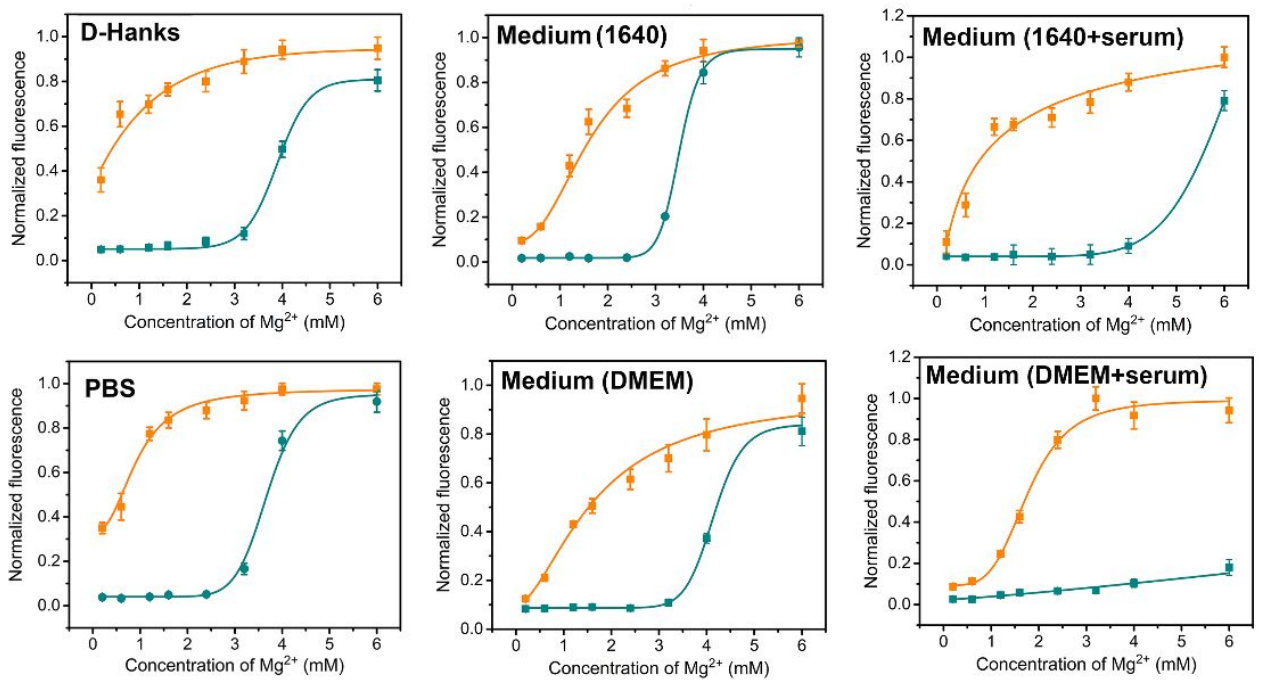

Figure S6. Fluorescence signal variation of exponential sFiR in equilibrium buffer and medium with different concentration of magnesium ions. Error bars represent the standard deviation of three experiments. 
A

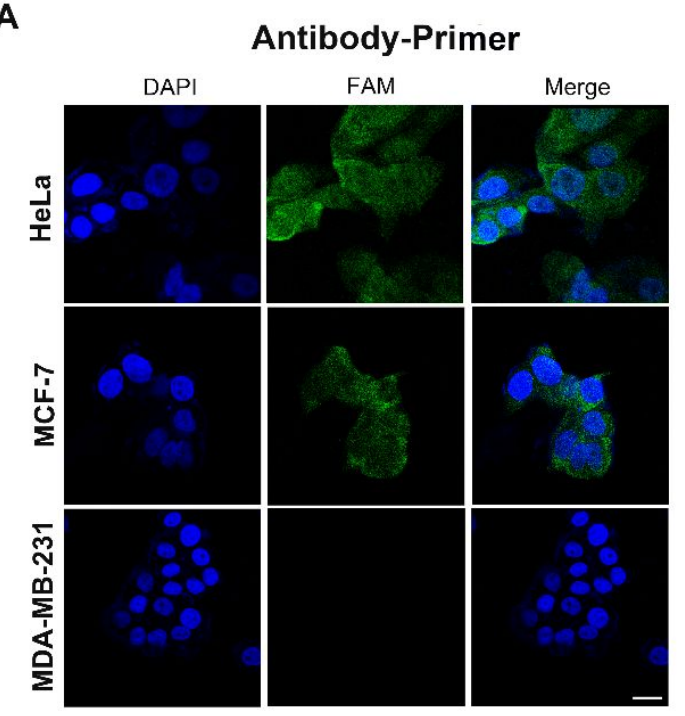

B Immunassay

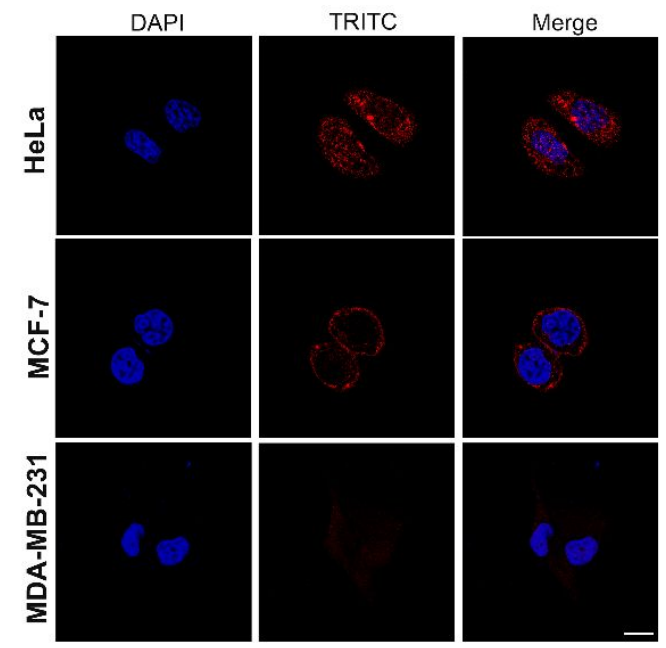

Figure S7. Immunofluorescence of JUP in three cell lines (A) direct assay (B) indirect assay. Scale: $20 \mu \mathrm{m}$. 

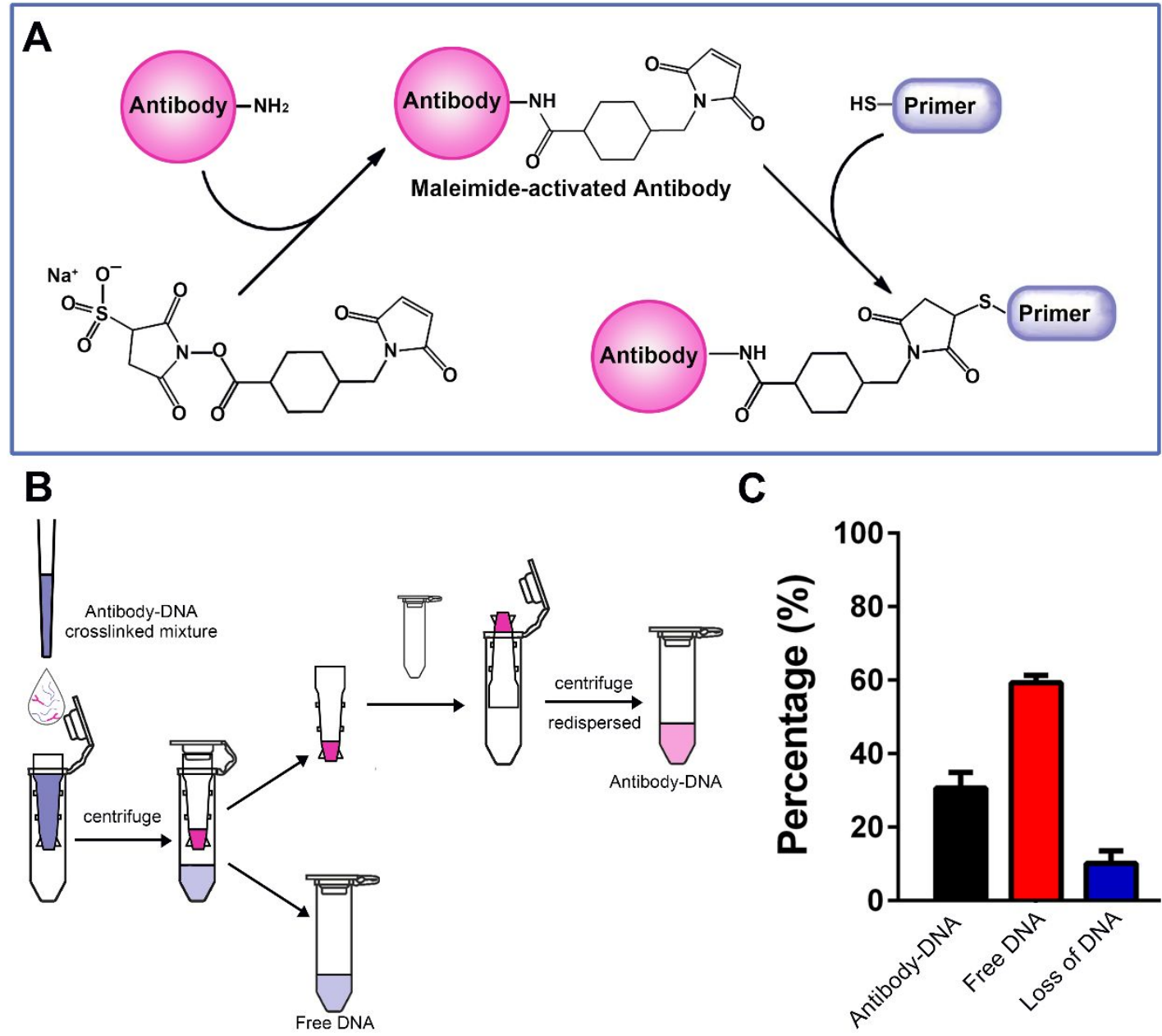

C

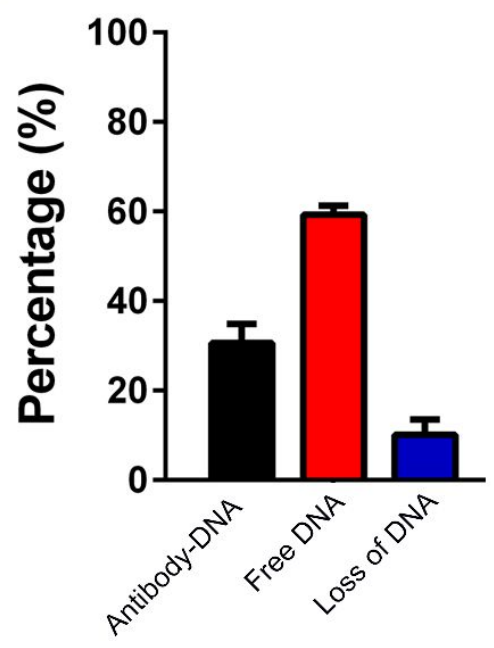

Figure S8. Crosslinking efficiency between the antibody and the primer. (A) Schematic diagram of crosslink between antibody and primer. (B) Schematic diagram of purification of the crosslinked JUP antibody-primer. (C) Study on the crosslinking efficiency between the antibody and the primer. Error bars represent the standard deviation of three experiments. 

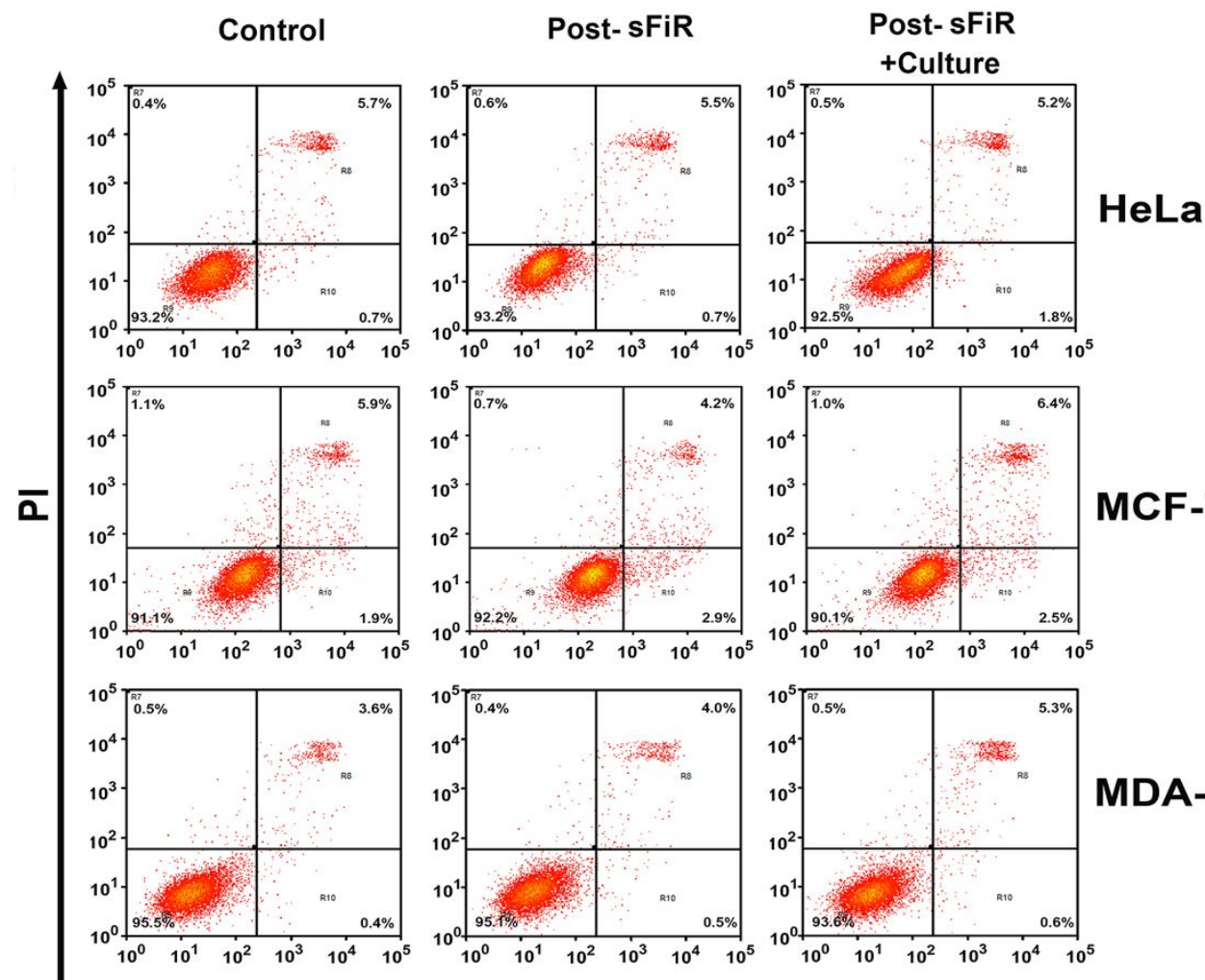

\section{Annexin V-Kfluo647}

Figure S9. Apoptosis analysis of different cells before and after immuno-sFiR analysis.

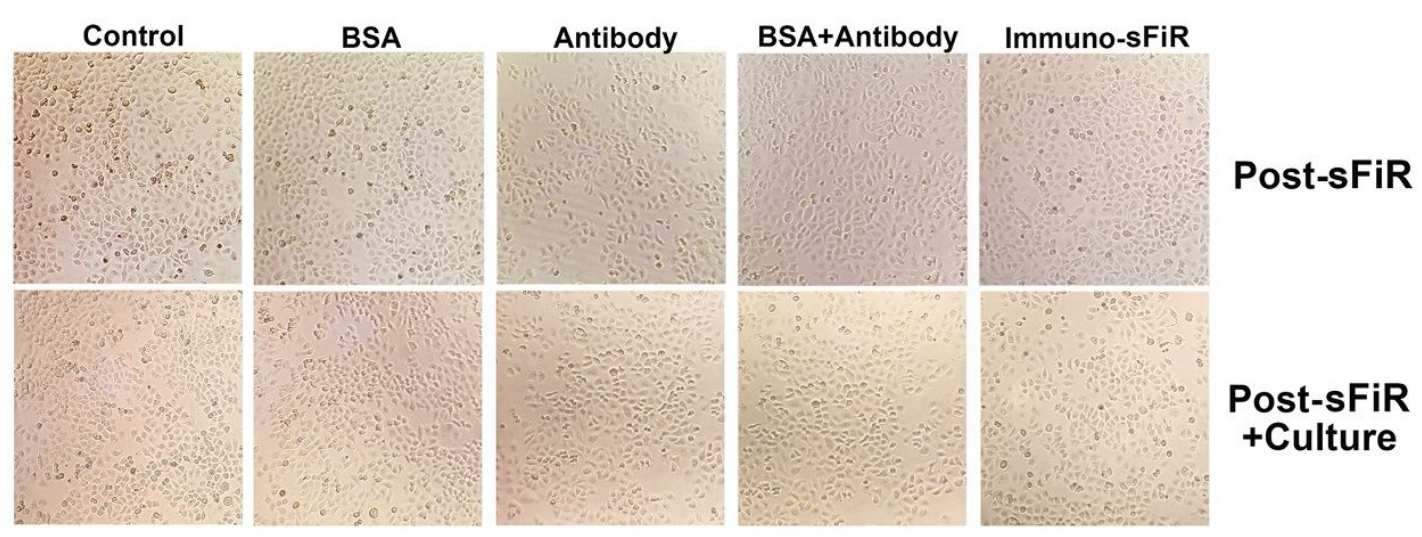

Figure S10. Morphology of HeLa cells after immuno-sFiR analysis. 


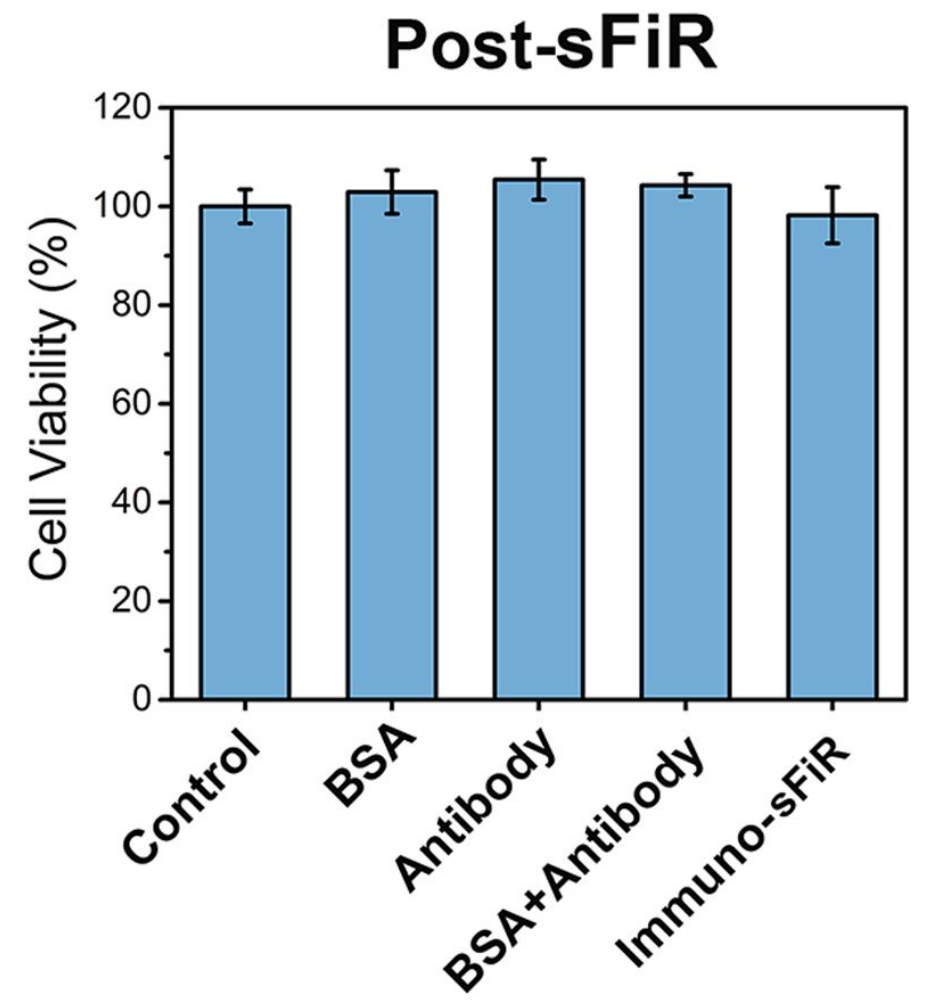

Figure S11. Cell viability analysis of HeLa cells after immuno-sFiR analysis.

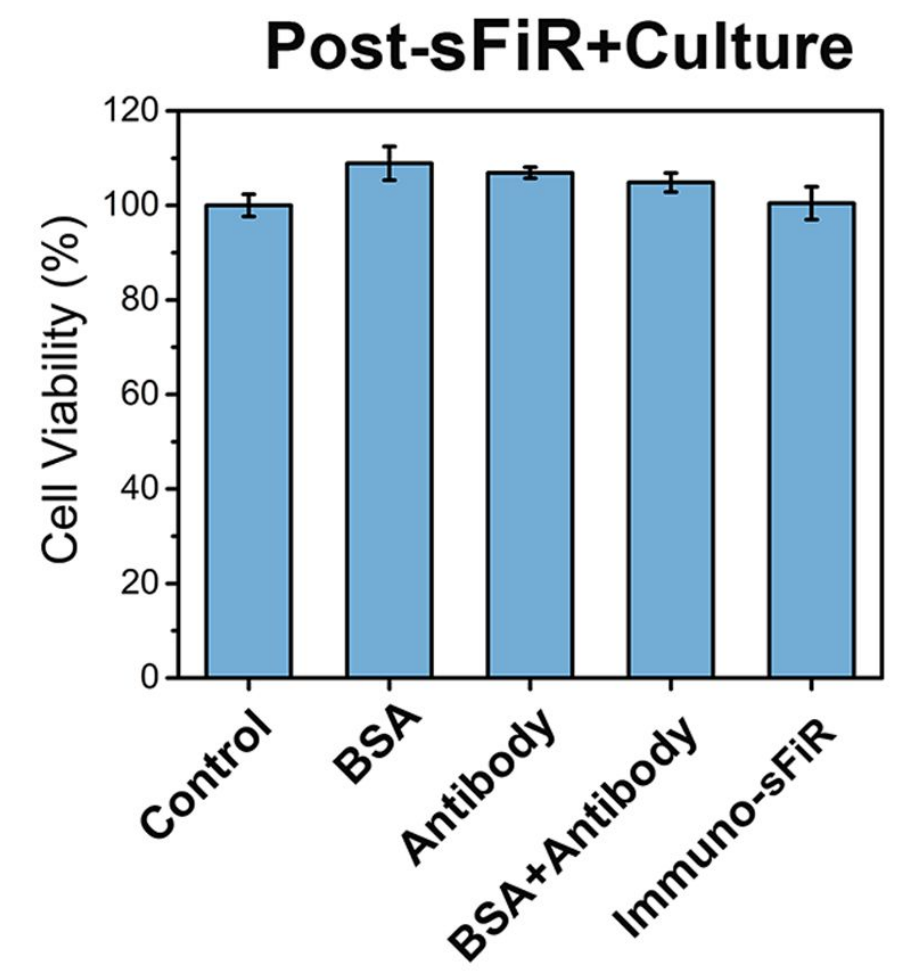

Figure S12. Cell viability analysis of HeLa cells with immuno-sFiR and continued 
culture after the analysis.

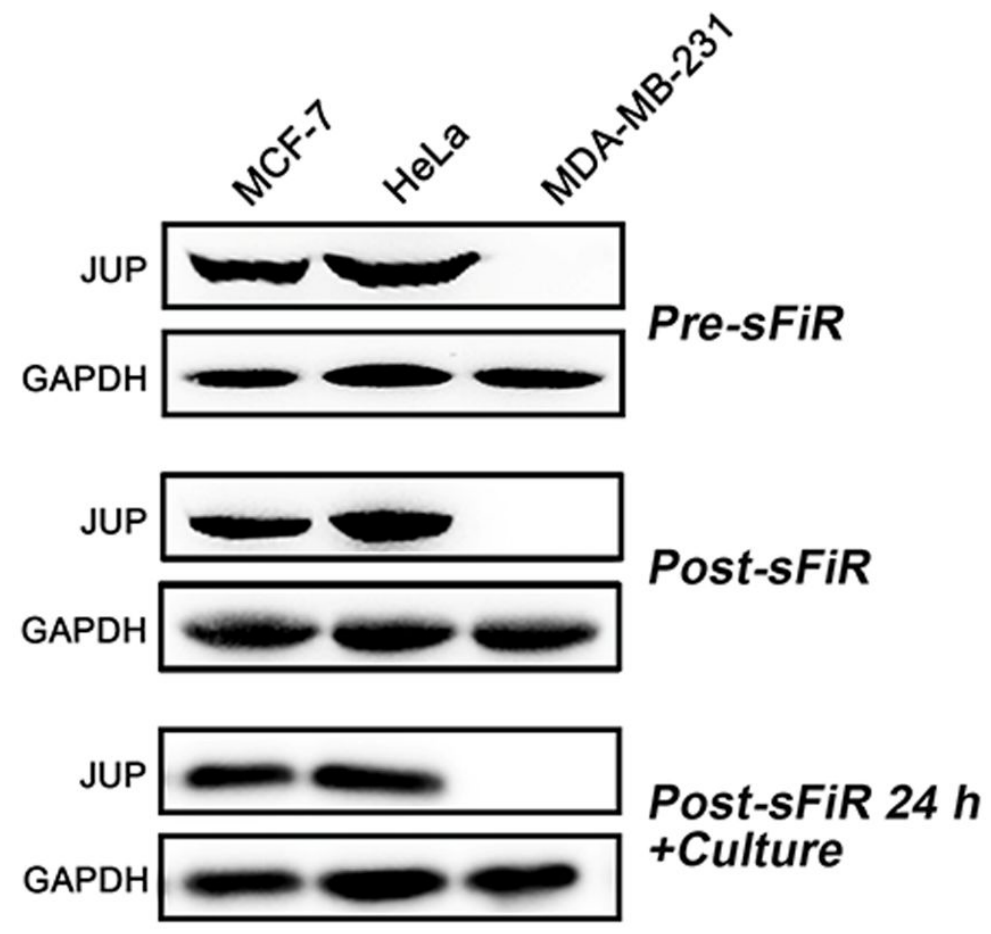

Figure S13. JUP expression before and after immuno-sFiR analysis.

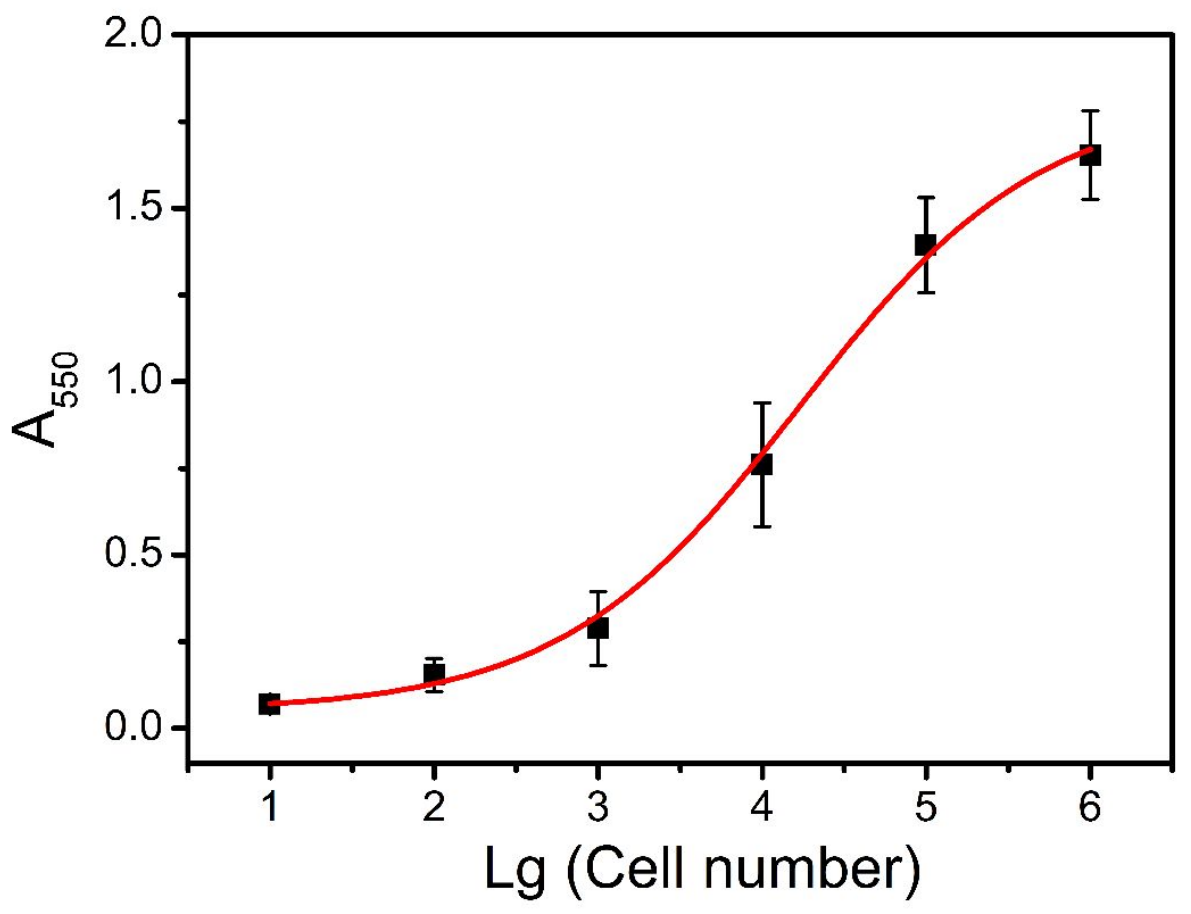


Figure S14. Standard curve at different concentration of HeLa cells using MTT assay. Error bars represent the standard deviation of three experiments. 